\title{
Gaussian and plane-wave mixed density fitting for periodic systems
}

Qiming Sun, Timothy C. Berkelbach, James D. McClain, and Garnet Kin-Lic Chan

Citation: The Journal of Chemical Physics 147, 164119 (2017);

View online: https://doi.org/10.1063/1.4998644

View Table of Contents: http://aip.scitation.org/toc/jcp/147/16

Published by the American Institute of Physics

\section{AlP|The Journal of Chemical Physics}




\title{
Gaussian and plane-wave mixed density fitting for periodic systems
}

\author{
Qiming Sun, ${ }^{1}$ Timothy C. Berkelbach, ${ }^{2}$ James D. McClain, ${ }^{1}$ and Garnet Kin-Lic Chan ${ }^{1, a)}$ \\ ${ }^{1}$ Division of Chemistry and Chemical Engineering, California Institute of Technology, Pasadena, \\ California 91125, USA \\ ${ }^{2}$ Department of Chemistry and James Franck Institute, University of Chicago, Chicago, Illinois 60637, USA
}

(Received 2 August 2017; accepted 15 October 2017; published online 31 October 2017)

We introduce a mixed density fitting scheme that uses both a Gaussian and a plane-wave fitting basis to accurately evaluate electron repulsion integrals in crystalline systems. We use this scheme to enable efficient all-electron Gaussian based periodic density functional and Hartree-Fock calculations. Published by AIP Publishing. https://doi.org/10.1063/1.4998644

\section{INTRODUCTION}

Computing the two-electron repulsion integrals (ERIs)

$$
(\mu \nu \mid \kappa \lambda)=\int \mu^{*}\left(\mathbf{r}_{1}\right) v\left(\mathbf{r}_{1}\right) \frac{1}{r_{12}} \kappa^{*}\left(\mathbf{r}_{2}\right) \lambda\left(\mathbf{r}_{2}\right) d \mathbf{r}_{1} \mathbf{r}_{2}
$$

has been a traditional bottleneck in electronic structure modeling when using a Gaussian basis. The ERIs serve as final targets of computation or may be used in contractions to form intermediates, such as in the Coulomb and exchange operators in mean-field calculations.

Various approximations have been proposed to reduce the cost of ERI computation and their associated intermediates in both molecular and crystalline systems. Many of them, including Gaussian density fitting (GDF), ${ }^{1-15}$ Cholesky decomposition, ${ }^{16-18}$ plane-wave Fourier transform techniques, ${ }^{19-23}$ and the pseudo-spectral method and its variants, ${ }^{24,25}$ can be considered to fall under the general rubric of density fitting (DF) methods. Density fitting can be used both when computing individual ERIs and in intermediate formation. The basic idea is to approximate the two-center atomic orbital pair density in Eq. (1) with an expansion in auxiliary functions, the fitting basis. The approximate density $\rho$ ' is obtained by minimizing its distance to the reference two-center density $\rho$ with respect to a metric $g\left(\mathbf{r}_{1}, \mathbf{r}_{2}\right)$ [such as the Coulomb metric $r_{12}^{-1}$ or overlap metric $\left.\delta\left(\mathbf{r}_{1}-\mathbf{r}_{2}\right)\right]$

$$
\min _{\rho^{\prime}} \iint\left[\rho\left(\mathbf{r}_{1}\right)-\rho^{\prime}\left(\mathbf{r}_{1}\right)\right] g\left(\mathbf{r}_{1}, \mathbf{r}_{2}\right)\left[\rho\left(\mathbf{r}_{2}\right)-\rho^{\prime}\left(\mathbf{r}_{2}\right)\right] d \mathbf{r}_{1} d \mathbf{r}_{2} .
$$

By choosing different metrics and fitting bases, one recovers the different schemes mentioned above. However, the most common version of DF uses a Gaussian fitting basis in conjunction with the Coulomb metric. We will refer to this standard combination of fitting basis and Coulomb metric as Gaussian density fitting (GDF). Gaussian density fitting is available in almost all the major quantum chemistry packages today. ${ }^{3-5,26-34}$

In this work, we extend the Gaussian DF methodology to a mixed basis density fitting (MDF). This creates an efficient DF

a)Electronic mail: gkc1000@gmail.com framework well suited to the all-electron modeling of periodic systems. The basic idea in MDF is to use a mixed auxiliary basis of Gaussians $\chi_{Q}(\mathbf{r})$ and plane-waves (PWs), expanding the density as

$$
\rho(\mathbf{r})=\sum_{Q} \chi_{Q}(\mathbf{r}) d_{Q}+\sum_{\mathbf{G}} e^{i \mathbf{G} \cdot \mathbf{r}} c_{\mathbf{G}} .
$$

The mixed-basis form allows the representation of compact densities through the Gaussian functions $\chi_{Q}(\mathbf{r})$, while offering systematic convergence for smooth densities through the PWs. These two properties address the challenges of Coulomb evaluation in all-electron periodic calculations, where contributions from both the core and diffuse interstitial densities must be efficiently computed. Further, the use of a PW representation provides a natural way to handle the Coulomb divergence that appears in periodic settings. Although such all-electron calculations can be expected to be more expensive than pseudo-potential calculations, they allow us to carry out computations free of pseudo-potential error.

There are related works in the literature. These include the Gaussian and (augmented) plane-wave formalism by Parrinello and co-workers ${ }^{21,35,36}$ and the Fourier transform Coulomb method of Füsti-Molnar and Pulay. ${ }^{19,37}$ In both of these, Gaussian basis sets are used to expand the orbitals, and the density matrix contributions of Gaussians with large exponents (compact Gaussians) and small exponents (smooth Gaussians) are separated. The Coulomb potential and energy contributions of the smooth Gaussians are evaluated by PW density fitting using the fast Fourier transform (FFT), while the compact Gaussian ERIs are evaluated explicitly. Thus, unlike in our mixed density fitting, Gaussian density fitting is not used at all. Further, both works are concerned with optimizing the evaluation of the Coulomb potential and energy only, rather than the more general ERI kernel, as used in the computation of exchange and in many-body methods. Some other differences include the manner in which compact and smooth densities are partitioned, as well as our use of analytical Fourier transforms (AFT) to achieve higher accuracy than the FFT with the same number of PWs. The impact of these choices will become apparent in the benchmark applications discussed below. 
The rest of this manuscript describes in detail the implementation of the mixed density fitting scheme and its benchmarking. In Secs. II A and II B, we present the formulae to compute the 4-index ERIs in terms of the MDF basis. The procedure to carry out GDF in a periodic system, which serves as a comparison for MDF, is discussed in Sec. II C using some formulae developed in the MDF framework. The MDF scheme is benchmarked for the all-electron Coulomb, exchange, and total energy at the Hartree-Fock level, and the all-electron band structure at the density functional level, for some simple crystals in Sec. III. Our conclusions are presented in Sec. IV.

\section{THEORY}

\section{A. Mixed density fitting method for periodic systems}

In an $N$-cell crystalline system, the AO functions $\phi_{\mu}^{\mathbf{k}}(\mathbf{r})$ are translational-symmetry-adapted linear combinations of Gaussian atomic orbitals $\mu(\mathbf{r}),{ }^{38}$

$$
\phi_{\mu}^{\mathbf{k}}(\mathbf{r})=\sum_{\mathbf{T}} e^{i \mathbf{k} \cdot \mathbf{T}} \mu(\mathbf{r}-\mathbf{T}),
$$

where $\mathbf{T}$ is a translation vector and $\mathbf{k}$ is a crystal momentum vector. In the mixed density fitting scheme, the AO products $\rho_{\mu \nu}(\mathbf{r})$ are approximated by an expansion of periodic Gaussian fitting functions plus plane-wave functions,

$$
\begin{aligned}
\rho_{\mu \nu}(\mathbf{r})= & \phi_{\mu}^{\mathbf{k}_{\mu} *}(\mathbf{r}) \phi_{\nu}^{\mathbf{k}_{\nu}}(\mathbf{r})=\sum_{Q} \phi_{Q}^{\mathbf{k}_{\mu \nu}}(\mathbf{r}) d_{Q, \mu \nu} \\
& +\sum_{\mathbf{G}+\mathbf{k}_{\mu \nu} \neq 0} \frac{e^{i\left(\mathbf{G}+\mathbf{k}_{\mu \nu}\right) \cdot \mathbf{r}}}{\sqrt{N \Omega}} c_{\mathbf{G}, \mu \nu}+\bar{\rho}_{\mu \nu},
\end{aligned}
$$

where $\mathbf{k}_{\mu \nu}=-\mathbf{k}_{\mu}+\mathbf{k}_{v}, N \Omega$ is the total volume of the computational crystal, $\Omega$ is the volume of the unit cell, and $\bar{\rho}_{\mu \nu}$ is a constant that will be defined later. The fitting function $\phi_{Q}^{\mathbf{k}}(\mathbf{r})$ is defined as

$$
\begin{aligned}
\phi_{Q}^{\mathbf{k}}(\mathbf{r}) & =\chi_{Q}^{\mathbf{k}}(\mathbf{r})-\xi_{Q}^{\mathbf{k}}(\mathbf{r}) \\
& =\frac{1}{\sqrt{N}} \sum_{\mathbf{T}} e^{i \mathbf{k} \cdot \mathbf{T}}\left[\chi_{Q}(\mathbf{r}-\mathbf{T})-\xi_{Q}(\mathbf{r}-\mathbf{T})\right], \\
\chi_{Q}^{\mathbf{k}}(\mathbf{r}) & =\frac{1}{\sqrt{N}} \sum_{\mathbf{T}} e^{i \mathbf{k} \cdot \mathbf{T}} \chi_{Q}(\mathbf{r}-\mathbf{T}), \\
\xi_{Q}^{\mathbf{k}}(\mathbf{r}) & =\frac{1}{\sqrt{N}} \sum_{\mathbf{T}} e^{i \mathbf{k} \cdot \mathbf{T}} \xi_{Q}(\mathbf{r}-\mathbf{T}),
\end{aligned}
$$

where $\chi_{Q}(\mathbf{r})$ is a compact Gaussian fitting function and $\xi_{Q}(\mathbf{r})$ is a smooth Gaussian fitting function that is subtracted from it to ensure that the fitting basis functions carry zero net charge and zero multipoles. For example, for a $p$-type auxiliary function, we require that the dipole integral vanishes

$$
\int \mathbf{r}\left[\chi_{p}(\mathbf{r})-\xi_{p}(\mathbf{r})\right] d \mathbf{r}=0
$$

The Coulomb potential of a zero-charge and zero-multipole density decays exponentially in real space, and this allows us to compute the Coulomb integrals of the Gaussian fitting functions using lattice summation. Another choice of the fitting functions that can rapidly converge the Coulomb integrals with lattice summation is the momentless Poisson type orbitals ${ }^{13,30}$
(PTO). However, a small set of regular Gaussians (with multipole moment) is usually required for the PTO density fitting scheme to describe density in the local domain. The integrals for the regular Gaussians can be calculated using the approach we presented here.

The compensating function $\xi_{Q}(\mathbf{r})$ does not hold any other physical significance but should be chosen to be smooth so that its contributions can be efficiently compensated for in the PW expansion. Given a real space lattice sum truncation distance, the smoothness of $\xi_{Q}(\mathbf{r})$ can be optimized in the same manner as is done in the optimization of the Ewald parameter. ${ }^{39,40}$ Because the charge is excluded from the Gaussian fitting basis, we handle it as part of the PW expansion, and this is the last term in Eq. (4) (corresponding to $\mathbf{G}=0$ and $\mathbf{k}_{\mu}=\mathbf{k}_{v}$ ),

$$
\bar{\rho}_{\mu v}=\frac{1}{N \Omega} \int \phi_{\mu}^{\mathbf{k}_{\mu} *}(\mathbf{r}) \phi_{v}^{\mathbf{k}_{v}}(\mathbf{r}) d \mathbf{r}=\frac{S_{\mu v}}{\Omega},
$$

where

$$
S_{\mu \nu}=\sum_{\mathbf{T}} e^{i \mathbf{k}_{\nu} \cdot \mathbf{T}} \int \mu^{*}(\mathbf{r}) v(\mathbf{r}-\mathbf{T}) d \mathbf{r}
$$

is the AO overlap integral (per unit cell).

The fitting coefficients are obtained by minimizing the density fitting error in the Coulomb metric. This leads to a linear equation for the coefficients $d_{Q, \mu \nu}$ and $c_{\mathbf{G}, \mu \nu}$,

$$
\begin{array}{r}
\left(\begin{array}{ll}
\left(\phi_{P}^{-\mathbf{k}_{\mu \nu}} \mid \phi_{Q}^{\mathbf{k}_{\mu \nu}}\right) & \frac{4 \pi \rho_{P}\left(-\mathbf{G}-\mathbf{k}_{\mu \nu}\right)}{\sqrt{\Omega}\left|\mathbf{G}+\mathbf{k}_{\mu \nu}\right|^{2}} \\
\frac{4 \pi \rho_{Q}\left(\mathbf{G}+\mathbf{k}_{\mu \nu}\right)}{\sqrt{\Omega}\left|\mathbf{G}+\mathbf{k}_{\mu \nu}\right|^{2}} & \frac{4 \pi}{\left|\mathbf{G}+\mathbf{k}_{\mu \nu}\right|^{2}}
\end{array}\right)\left(\begin{array}{c}
d_{Q, \mu \nu} \\
c_{\mathbf{G}, \mu \nu}
\end{array}\right) \\
\quad=\left(\begin{array}{c}
\sqrt{N}\left[\left(\phi_{P}^{-\mathbf{k}_{\mu \nu}} \mid \phi_{\mu}^{\mathbf{k}_{\mu} *} \phi_{\nu}^{\mathbf{k}_{\nu}}\right)-\bar{V}_{P}^{-\mathbf{k}_{\mu \nu}} \bar{\rho}_{\mu \nu}\right] \\
\frac{4 \pi \rho_{\mu \nu}\left(\mathbf{G}+\mathbf{k}_{\mu \nu}\right)}{\sqrt{\Omega}\left|\mathbf{G}+\mathbf{k}_{\mu \nu}\right|^{2}}
\end{array}\right),
\end{array}
$$

where the integrals (derived in Appendix A) are

$$
\begin{aligned}
& \left(\phi_{P}^{-\mathbf{k}} \mid \phi_{Q}^{\mathbf{k}}\right)=\sum_{\mathbf{T}} e^{i \mathbf{k} \cdot \mathbf{T}} \int\left[\chi_{P}\left(\mathbf{r}_{1}\right)-\xi_{P}\left(\mathbf{r}_{1}\right)\right] \frac{1}{r_{12}} \\
& \times\left[\chi_{Q}\left(\mathbf{r}_{2}-\mathbf{T}\right)-\xi_{Q}\left(\mathbf{r}_{2}-\mathbf{T}\right)\right] d \mathbf{r}_{1} d \mathbf{r}_{2}, \\
& \rho_{Q}(\mathbf{G}+\mathbf{k})=\int e^{-i(\mathbf{G}+\mathbf{k}) \cdot \mathbf{r}}\left[\chi_{Q}(\mathbf{r})-\xi_{Q}(\mathbf{r})\right] d \mathbf{r}, \\
& \bar{V}_{P}^{\mathbf{k}}=\left\{\begin{array}{ll}
\frac{\pi}{\alpha_{P_{\xi}}}-\frac{\pi}{\alpha_{P_{\chi}}} & \mathbf{k}=0 \text { and } \chi_{P} \in \text { s-type GTOs } \\
0 & \text { otherwise }
\end{array},\right. \\
& \left(\phi_{P}^{-\mathbf{k}_{\mu \nu}} \mid \phi_{\mu}^{\mathbf{k}_{\mu} *} \phi_{\nu}^{\mathbf{k}_{\nu}}\right)=\sum_{\mathbf{T}_{\mu} \mathbf{T}_{\nu}} e^{i \mathbf{k}_{\nu} \cdot \mathbf{T}_{\nu}-i \mathbf{k}_{\mu} \cdot \mathbf{T}_{\mu}} \int\left[\chi_{P}\left(\mathbf{r}_{1}\right)-\xi_{P}\left(\mathbf{r}_{1}\right)\right] \\
& \times \frac{1}{r_{12}} \mu^{*}\left(\mathbf{r}_{2}-\mathbf{T}_{\mu}\right) v\left(\mathbf{r}_{2}-\mathbf{T}_{v}\right) d \mathbf{r}_{1} d \mathbf{r}_{2},
\end{aligned}
$$

$$
\rho_{\mu \nu}\left(\mathbf{G}+\mathbf{k}_{\mu \nu}\right)=\sum_{\mathbf{T}} e^{i \mathbf{k}_{\nu} \cdot \mathbf{T}} \int e^{-i\left(\mathbf{G}+\mathbf{k}_{\mu \nu}\right) \cdot \mathbf{r}} \mu^{*}(\mathbf{r}) v(\mathbf{r}-\mathbf{T}) d \mathbf{r} .
$$

In Eq. (14), $\alpha_{P_{\chi}}$ and $\alpha_{P_{\xi}}$ are the exponents of the Gaussian functions $\chi_{P}(\mathbf{r})$ and $\xi_{P}(\mathbf{r})$. In the above integrals, computing the three center integral (15) is demanding due to the double lattice sum, with a cost of $O\left(n^{2} m N_{c}^{2}\right)$ where $n$ is the number 
of AOs, $m$ is the number of auxiliary Gaussian functions, and $N_{c}$ is the number of images in the lattice summation.

Equations (13) and (16) involve the Fourier transforms of the fitting Gaussians and AO products. While one can approximate these integrals using a discrete fast Fourier transform (FFT), this is only practical if the Gaussians involved are not very steep, as for example, in pseudo-potential calculations; otherwise prohibitively large Fourier grids are necessary (see Sec. III). Alternatively, the integrals can be calculated analytically. The formulae for the analytical Fourier transforms are documented in Appendix B. The leading computational cost is for the AO products, which have a formal scaling of $O\left(n^{2} N_{G} N_{c}\right)$ where $N_{G}$ is the number of PWs. Although there is only one factor of $N_{c}$ (compared with the three center Gaussian integrals), the analytical Fourier transforms also become expensive for a large number of PWs. However, as long as the Gaussian fitting functions of core and valence characters are appropriately tuned, it is not difficult to require only a modest number of PWs in the MDF expansion of the smooth part of the density. The analytical Fourier transform technique can be used in both pseudo-potential and all-electron calculations. As the PWs in this approach are strictly used only to represent the density and not to numerically sample the Gaussians, one can use fewer PWs with the analytical Fourier transform than in a typical FFT-driven calculation.

Finally, when defining ERIs in a periodic system, we remove the net charge of the $\mathrm{AO}$ product to avoid the divergent Coulomb contribution, corresponding to removing the $\mathbf{G}=0$ singularity when $\mathbf{k}_{\mu \nu}=0 .{ }^{41}$ (The $\mathbf{G}=0$ electronic contribution, which only depends on the number of electrons in the unit cell, is appropriately handled together with the electronnuclear and nuclear-nuclear $\mathbf{G}=0$ contributions, yielding an additive constant to the total energy. ${ }^{41}$ ) Using the quantities defined in the MDF expansion, the periodic ERI (here, and in the following text, per unit cell) $W_{\mu \nu, \kappa \lambda}$ is assembled as

$$
\begin{aligned}
W_{\mu \nu, \kappa \lambda} & =\frac{1}{N} \int \frac{\left[\phi_{\mu}^{\mathbf{k}_{\mu} *}\left(\mathbf{r}_{1}\right) \phi_{\nu}^{\mathbf{k}_{v}}\left(\mathbf{r}_{1}\right)-\bar{\rho}_{\mu \nu}\right]\left[\phi_{\kappa}^{\mathbf{k}_{\kappa} *}\left(\mathbf{r}_{2}\right) \phi_{\lambda}^{\mathbf{k}_{\lambda}}\left(\mathbf{r}_{2}\right)-\bar{\rho}_{\kappa \lambda}\right]}{r_{12}} d \mathbf{r}_{1} d \mathbf{r}_{2} \\
& =\sum_{Q} \frac{d_{Q, \mu v}}{\sqrt{N}}\left(\phi_{Q}^{\mathbf{k}_{\mu \nu}} \mid \phi_{\kappa}^{\mathbf{k}_{\kappa} *} \phi_{\lambda}^{\mathbf{k}_{\lambda}}\right)-\bar{\rho}_{\kappa \lambda} \sum_{Q} \frac{d_{Q, \mu \nu}}{\sqrt{N}} \bar{V}_{Q}^{\mathbf{k}_{\mu \nu}}+\sum_{\mathbf{G}+\mathbf{k}_{\mu \nu} \neq 0} c_{\mathbf{G}, \mu \nu} \rho_{\kappa \lambda}\left(-\mathbf{G}+\mathbf{k}_{\kappa \lambda}\right) .
\end{aligned}
$$

In the ERI expression, crystal momentum conservation is used,

$$
\left(-\mathbf{k}_{\mu}+\mathbf{k}_{v}-\mathbf{k}_{\kappa}+\mathbf{k}_{\lambda}\right) \cdot \mathbf{a}=2 n \pi
$$

where $\mathbf{a}$ is the lattice vector.

\section{B. Linear dependence in the MDF fitting basis}

In the mixed fitting basis, the periodic Gaussian functions and PWs may become linearly dependent with respect to each other as each subset becomes increasingly complete. In practice, this causes numerical instabilities when solving the linear equation in the form (11) directly. To remove the linear dependencies, we orthogonalize the fitting functions with respect to the Coulomb metric through the transformation

$$
\left(\phi_{Q}^{\mathbf{k}}(\mathbf{r}) \frac{e^{i(\mathbf{G}+\mathbf{k}) \cdot \mathbf{r}}}{\sqrt{N \Omega}}\right)\left(\begin{array}{cc}
\mathbf{t}^{\mathbf{k}} & 0 \\
-\frac{\rho_{Q}(\mathbf{G}+\mathbf{k})}{\sqrt{\Omega}} \mathbf{t}^{\mathbf{k}} & \frac{|\mathbf{G}+\mathbf{k}|}{2 \sqrt{\pi}}
\end{array}\right),
$$

where the rectangular matrix $\mathbf{t}^{\mathbf{k}}$ is the transformation to diagonalize the dressed Coulomb matrix of the Gaussian fitting functions

$$
\begin{gathered}
\tilde{J}_{P Q}^{\mathbf{k}}=\left(\phi_{P}^{-\mathbf{k}} \mid \phi_{Q}^{\mathbf{k}}\right)-\sum_{\mathbf{G}+\mathbf{k} \neq 0} \frac{4 \pi \rho_{P}(-\mathbf{G}-\mathbf{k}) \rho_{Q}(\mathbf{G}+\mathbf{k})}{\Omega|\mathbf{G}+\mathbf{k}|^{2}}, \\
\mathbf{t}^{\mathbf{k} \dagger} \tilde{J}^{\mathbf{k}} \mathbf{t}^{\mathbf{k}}=\mathbf{1} .
\end{gathered}
$$

Although different choices can be made to remove linear dependencies, different schemes do not share the same numerical stability. We used the transformation (19) because it does not mix Gaussian functions into the PWs. An advantage of the PW basis is that the Coulomb operator is diagonal in the PW representation. Manipulating the basis orthogonalization in this diagonal representation is straightforward and numerically stable, leading to the normalization factor $|\mathbf{G}+\mathbf{k}| / 2 \sqrt{\pi}$ in Eq. (19). Projecting the PWs out of the Gaussian functions in (20) leads to a highly singular matrix. To remove the linear dependence of the Gaussian functions, we diagonalize this singular matrix and remove the eigenvectors associated with small eigenvalues below a threshold. The effect of the linear dependence threshold on the stability of the results is tested in Sec. III. In our program, we use a default threshold of $10^{-9}$.

Applying transformation (19) to the linear equation (11) followed by the removal of small eigenvalues allows us to stably determine the density fitting coefficients. With respect to the transformed fitting functions, we can define a new MDF expression for the ERIs in (17),

$$
\begin{aligned}
W_{\mu \nu, \kappa \lambda}= & \sum_{i} L_{i, \mu \nu} L_{i, \kappa \lambda} \\
& +\sum_{\mathbf{G}+\mathbf{k}_{\mu \nu} \neq 0} \frac{4 \pi \rho_{\mu \nu}\left(\mathbf{G}+\mathbf{k}_{\mu \nu}\right) \rho_{\kappa \lambda}\left(-\mathbf{G}+\mathbf{k}_{\kappa \lambda}\right)}{\Omega\left|\mathbf{G}+\mathbf{k}_{\mu \nu}\right|^{2}}, \\
L_{i, \mu v}= & \sum_{P} t_{P i}^{\mathbf{k}_{\mu \nu} *}\left[\left(\phi_{P}^{-\mathbf{k}_{\mu v}} \mid \phi_{\mu}^{\mathbf{k}_{\mu} *} \phi_{\nu}^{\mathbf{k}_{v}}\right)-\bar{V}_{P}^{-\mathbf{k}_{\mu v}} \bar{\rho}_{\mu \nu}\right. \\
& \left.-\sum_{\mathbf{G}+\mathbf{k}_{\mu \nu} \neq 0} \frac{4 \pi \rho_{P}\left(-\mathbf{G}-\mathbf{k}_{\mu \nu}\right) \rho_{\mu \nu}\left(\mathbf{G}+\mathbf{k}_{\mu \nu}\right)}{\Omega\left|\mathbf{G}+\mathbf{k}_{\mu \nu}\right|^{2}}\right] .
\end{aligned}
$$




\section{Gaussian density fitting for periodic systems}

In the current work, we will benchmark mixed density fitting against standard Gaussian density fitting. We first describe how GDF may be efficiently implemented in periodic systems using some of the results introduced above for MDF. In the periodic setting, the AO products in the standard GDF method are expanded in a set of periodic Gaussian fitting functions,

$$
\rho_{\mu v}(\mathbf{r})=\sum_{Q} \chi_{Q}^{\mathbf{k}_{\mu v}}(\mathbf{r}) d_{Q, \mu \nu}
$$

The Coulomb metric when used directly in the periodic setting diverges. Thus, we exclude the net charge of the AO products in the fitting expansion

$$
\begin{array}{r}
\rho_{\mu \nu}(\mathbf{r})-\bar{\rho}_{\mu \nu}=\sum_{Q}\left(\chi_{Q}^{\mathbf{k}_{\mu \nu}}(\mathbf{r})-\bar{\chi}_{Q}^{\mathbf{k}_{\mu \nu}}\right) d_{Q, \mu v} . \\
\bar{\chi}_{Q}^{\mathbf{k}}=\left\{\begin{array}{l}
\frac{\sqrt{N}}{\Omega} \mathbf{k}=0 \text { and } \chi_{Q}(\mathbf{r}) \in s \text {-type GTOs } \\
0 \quad \text { otherwise }
\end{array}\right.
\end{array}
$$

The two-electron integrals can then be formulated in terms of the GDF quantities as

$$
\begin{gathered}
W_{\mu \nu, \kappa \lambda}=\sum_{P Q} \mathcal{V}_{P, \mu \nu}\left(\mathcal{J}^{-1}\right)_{P Q} \mathcal{V}_{Q, \kappa \lambda}, \\
\mathcal{V}_{P, \mu \nu}=\frac{1}{\sqrt{N}}\left(\chi_{P}^{\mathbf{k}_{\nu \mu}}-\bar{\chi}_{P}^{\mathbf{k}_{\nu \mu}} \mid \phi_{\mu}^{\mathbf{k}_{\mu} *} \phi_{\nu}^{\mathbf{k}_{v}}-\bar{\rho}_{\mu \nu}\right), \\
\mathcal{J}_{P Q}=\left(\chi_{P}^{\mathbf{k}_{\nu \mu}}-\bar{\chi}_{P}^{\mathbf{k}_{\nu \mu}} \mid \chi_{Q}^{\mathbf{k}_{\mu \nu}}-\bar{\chi}_{Q}^{\mathbf{k}_{\mu \nu}}\right) .
\end{gathered}
$$

The two-center and three-center Coulomb integrals represent Coulomb interactions between chargeless density distributions and thus are not divergent in the real space lattice summation; however, the convergence may be very slow or even conditional on the summation order. To accelerate the lattice summation, we can insert a compensating function $\xi^{\mathbf{k}}(\mathbf{r})$ in the density fitting expansion that removes higher multipoles of $\chi_{Q}^{\mathbf{k}}(\mathbf{r})$ as in the MDF procedure,

$$
\begin{aligned}
\rho_{\mu \nu}(\mathbf{r})-\bar{\rho}_{\mu \nu} & =\sum_{Q}\left(\chi_{Q}^{\mathbf{k}_{\mu \nu}}(\mathbf{r})-\xi_{Q}^{\mathbf{k}_{\mu \nu}}(\mathbf{r})+\xi_{Q}^{\mathbf{k}_{\mu \nu}}(\mathbf{r})-\bar{\chi}_{Q}^{\mathbf{k}_{\mu \nu}}\right) d_{Q, \mu \nu} \\
& =\sum_{Q} \phi_{Q}^{\mathbf{k}_{\mu v}}(\mathbf{r}) d_{Q, \mu \nu}+\sum_{Q}\left(\xi_{Q}^{\mathbf{k}_{\mu \nu}}(\mathbf{r})-\bar{\chi}_{Q}^{\mathbf{k}_{\mu \nu}}\right) d_{Q, \mu \nu}
\end{aligned}
$$

This allows us to efficiently compute the two-center and threecenter integrals in a two-step scheme: first we evaluate the integrals involving $\phi_{Q}(\mathbf{r})$ using real space lattice summation; then the remaining contributions are evaluated using a PW expansion. With this scheme, the integrals (28) and (29) are obtained as

$$
\begin{aligned}
\mathcal{V}_{P, \mu \nu}= & \left(\phi_{P}^{-\mathbf{k}_{\mu \nu}} \mid \phi_{\mu}^{\mathbf{k}_{\mu *} \phi_{\nu} \mathbf{k}_{\nu}}\right)-\bar{V}_{P}^{-\mathbf{k}_{\mu \nu}} \bar{\rho}_{\mu \nu} \\
& +\sum_{\mathbf{G}+\mathbf{k}_{\mu \nu} \neq 0} \frac{4 \pi \rho_{\xi P}\left(-\mathbf{G}-\mathbf{k}_{\mu \nu}\right) \rho_{\mu \nu}\left(\mathbf{G}+\mathbf{k}_{\mu \nu}\right)}{\Omega\left|\mathbf{G}+\mathbf{k}_{\mu \nu}\right|^{2}},
\end{aligned}
$$

$$
\begin{aligned}
\mathcal{J}_{P Q}= & \left(\phi_{P}^{-\mathbf{k}_{\mu \nu}} \mid \phi_{Q}^{\mathbf{k}_{\mu \nu}}\right) \\
& +\sum_{\mathbf{G}+\mathbf{k}_{\mu \nu} \neq 0} \frac{4 \pi \rho_{\xi_{P}}\left(-\mathbf{G}-\mathbf{k}_{\mu \nu}\right) \rho_{Q}\left(\mathbf{G}+\mathbf{k}_{\mu \nu}\right)}{\Omega\left|\mathbf{G}+\mathbf{k}_{\mu \nu}\right|^{2}}, \\
& +\sum_{\mathbf{G}+\mathbf{k}_{\mu \nu} \neq 0} \frac{4 \pi \rho_{P}\left(-\mathbf{G}-\mathbf{k}_{\mu \nu}\right) \rho_{\xi_{Q}}\left(\mathbf{G}+\mathbf{k}_{\mu \nu}\right)}{\Omega\left|\mathbf{G}+\mathbf{k}_{\mu \nu}\right|^{2}} \\
& +\sum_{\mathbf{G}+\mathbf{k}_{\mu \nu} \neq 0} \frac{4 \pi \rho_{\xi_{P}}\left(-\mathbf{G}-\mathbf{k}_{\mu \nu}\right) \rho_{\xi_{Q}}\left(\mathbf{G}+\mathbf{k}_{\mu \nu}\right)}{\Omega\left|\mathbf{G}+\mathbf{k}_{\mu \nu}\right|^{2}}
\end{aligned}
$$

where

$$
\rho_{\xi_{P}}(\mathbf{G}+\mathbf{k})=\int e^{-i(\mathbf{G}+\mathbf{k}) \cdot \mathbf{r}} \xi_{P}(\mathbf{r}) d \mathbf{r} .
$$

Note that in the GDF calculations, we always use sufficient number of $P W$ s to completely converge the $P W$ representation of the compensating Gaussian. This ensures that the GDF calculations are a measure purely of the quality of the original Gaussian density fitting basis.

\section{BENCHMARKING MDF}

We have implemented the MDF method as described above in our electronic structure program package PySCF. ${ }^{42}$ To test the accuracy of the MDF method, we first computed the $\Gamma$-point Hartree-Fock Coulomb $\left(E_{J}\right)$ and exchange energies $\left(E_{K}\right)$ for a system of hydrogen atoms in the diamond cubic crystal structure (eight atoms per cell, $\mathrm{Fd} \overline{3} \mathrm{~m}$ space group), with the lattice parameter $a=3.567 \AA$ and the cc-pVDZ basis. The MDF Gaussian fitting basis was the even tempered basis (ETB) $10 s 6 p 2 d$ (see Table I). The compensating Gaussians [see Eq. (5)] were chosen to have exponent 0.2 . The PW basis was constructed from a uniform reciprocal grid. The real-space lattice summation was truncated at a distance of $9.2 \AA$. This ensured that both the AO basis and auxiliary Gaussian basis lattice sums were fully converged.

We compare the different kinds of density fitting in Figs. 1(a) and 1(b). Using pure GDF and the large even tempered fitting basis, we can fit $E_{J}$ to roughly $0.1 \mathrm{~m} E_{\mathrm{h}}$ accuracy and $E_{K}$ to roughly $1 \mathrm{~m} E_{\mathrm{h}}$ accuracy. Note that the $\mathrm{H}$ atom cc-pVDZ basis does not contain any steep Gaussian functions. This means it is also practical in this system to use only PWs as the fitting functions. We show the results of PW density fitting (labeled FFT) where the PW coefficients and contributions are determined by FFT. The PW density fitting converges the Coulomb

TABLE I. Even-tempered basis, $\alpha \beta^{i}, i=0, \ldots, n-1$.

\begin{tabular}{lrcc}
\hline \hline Angular momentum & $n$ & $\alpha$ & $\beta$ \\
\hline H atom 10s6p2d & & & \\
$s$ & 10 & 0.244 & 1.6 \\
$p$ & 6 & 0.596 & 1.6 \\
$d$ & 2 & 1.454 & 1.6 \\
Si atom 20s16p13d7f2g & & & \\
$s$ & 20 & 0.333 & 1.8 \\
$p$ & 16 & 0.324 & 1.8 \\
$d$ & 13 & 0.316 & 1.8 \\
$F$ & 7 & 0.310 & 1.8 \\
$G$ & 2 & 0.550 & 1.8 \\
\hline \hline
\end{tabular}



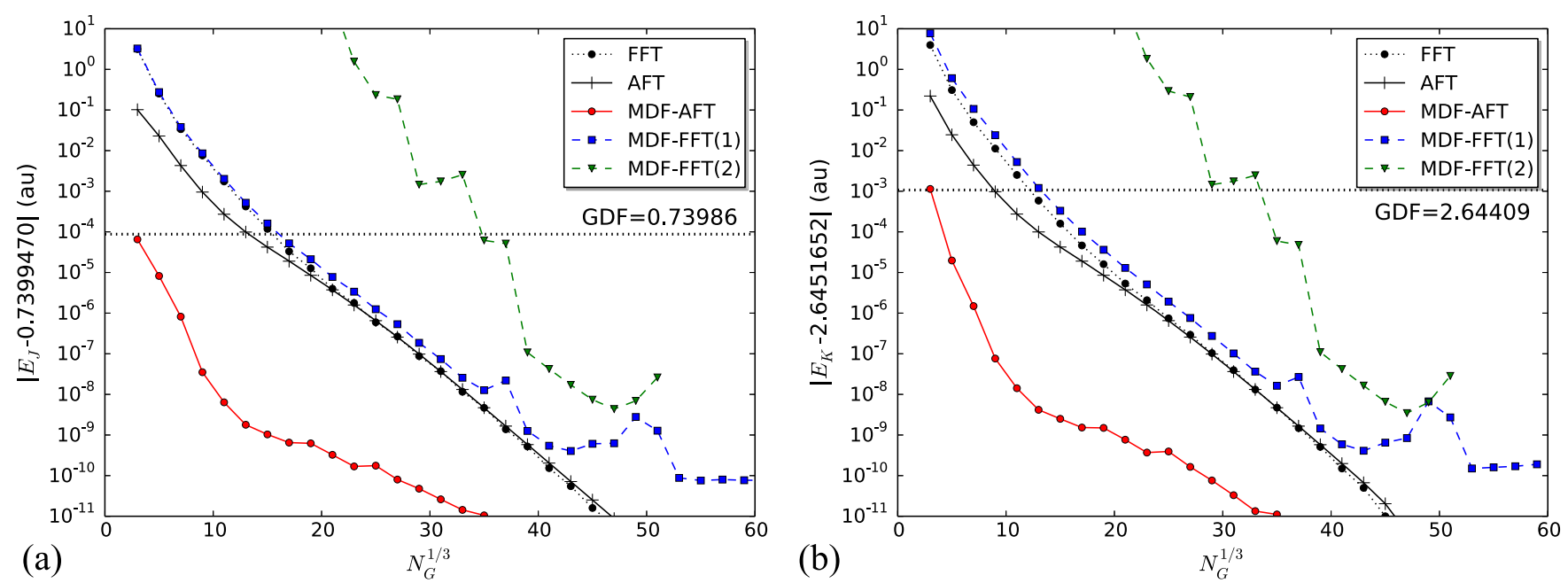

FIG. 1. Coulomb and exchange interactions per unit cell for the $\mathrm{H}$ crystal ( $\Gamma$ point). In the $\mathrm{H}$ crystal, the reference energies are computed using a pure PW fitting basis and FFT computation of all terms with $N_{G}^{1 / 3}=101$ grid points. The ETB basis $10 s 6 p 2 d$ is employed for MDF and GDF. (a) Coulomb energy for the H crystal. (b) Exchange energy for the $\mathrm{H}$ crystal.

and exchange energy very systematically as a function of the number of PWs. This demonstrates the strength of including PWs in the fitting basis, and in fact we use the systematic convergence to estimate the reference Coulomb and exchange energies. Finally, we observe the effect of using both Gaussians and PWs in the MDF expansion (labeled MDF-AFT). We see that introducing the Gaussian fitting basis leads to improved convergence relative to the pure $\mathrm{PW}$ expansion. The MDF expansion is 4-5 orders of magnitude more accurate than the pure PW expansion with the same number of PWs. The accuracy of GDF itself is close to the accuracy of MDF with a minimal $\mathrm{PW}$ basis (27 PWs, 3 grid points per direction). Since the difference between the Gaussian fitting basis in GDF and MDF is the set of compensating Gaussian functions in MDF, this reflects the fact that the compensating Gaussians used in MDF are here well represented by a small number of PWs. Further, adding a modest number of PWs in MDF significantly improves the accuracy over pure GDF, for example, $729 \mathrm{PWs}$ (9 per axis) reduce the fitting error to $0.1 \mu E_{\mathrm{h}}$.

In the MDF-AFT results, we used analytical Fourier transforms for all PW-related integrals in the MDF method. As discussed in Sec. II A, it is also possible to use the discrete FFT to compute these integrals although additional errors are expected. Note that there are three equations- (20), (22), and (23) - that involve quantities in reciprocal space. The FFT cannot be used to obtain the reciprocal space densities in Eq. (20) because the numerical FFT destroys the positive definiteness of the metric. We tested the use of the FFT integrals in the other two equations as follows: (1) using FFT reciprocal space quantities in the second term of Eq. (22), denoted MDF-FFT(1) in Figs. 1(a) and 1(b); (2) using FFT reciprocal space quantities in both Eqs. (22) and (23), denoted MDF-FFT(2). To illustrate the density sampling error when using the FFT, we also computed the PW related integrals using AFT, and the pure AFT results are also presented in Figs. 1(a) and 1(b). MDF-FFT(1) gives a similar error to pure PW density fitting (using FFT for the PW related integrals) because the errors from the FFT density sampling are larger than the corrections introduced by the Gaussian fitting functions in MDF. The error in MDF-FFT(2) is more severe as the numerical errors introduced by the FFT are compounded in Eqs. (22) and (23). In either case, the use of the FFT to approximate the quantities involved in MDF clearly leads to unacceptable errors and negates the advantages of the MDF scheme.

Figure 2 shows the convergence of $\Gamma$-point all-electron Hartree-Fock energies for the silicon crystal ( $\mathrm{Fd} \overline{3} \mathrm{~m}$ symmetry, lattice parameter $a=5.431 \AA$, cc-pVDZ basis). We used a large ETB fitting basis $20 s 16 p 13 d 7 f 2 g$ (see Table I). The exponents of the compensating Gaussians were set to 0.2 , and the real space lattice sums were truncated at $12 \AA$. We use this system to test the effect of the linear dependency threshold, and linear thresholds of $10^{-6}, 10^{-7}, \ldots, 10^{-14}$ were tested, keeping all other settings the same. For clarity, we only present the results of $10^{-9}, 10^{-10}, 10^{-11}$, and $10^{-14}$ in Fig. 2.

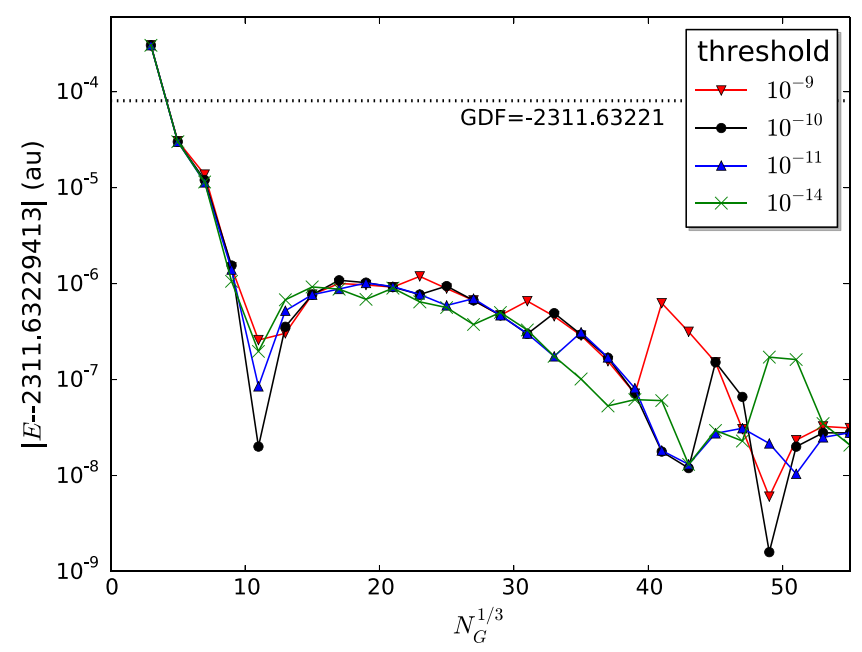

FIG. 2. Hartree-Fock energy per unit cell for the Si crystal. The reference energies are computed using MDF with $N_{G}^{1 / 3}=81$ grid points. The ETB basis is $20 s 16 p 13 d 7 f 2 g$. 
Because of the large Gaussian fitting basis, the GDF method achieves good accuracy for the total energy. This is close to the accuracy of the MDF method with 125 PWs (corresponding to a PW energy cutoff of $20 \mathrm{eV}$ ). In this system, the presence of core functions means that PWs alone are insufficient to expand the densities; however, when used in conjunction with Gaussians, MDF systematically improves beyond the GDF result. When the plane waves are augmented by Gaussians, the linear dependency between plane waves and Gaussians may lead to numerical stability issue. The optimal accuracy requires a reasonable linear dependency threshold. A threshold too tight would cause numerical instability while a threshold too loose would increase the basis set incompleteness error since many auxiliary functions are removed. For a small number of PWs, because the PWs only overlap the most diffuse auxiliary Gaussians, which has minor contributions to the HF energy, the tested linear dependence thresholds do not present significant differences. The bigger differences are found for 1331 PWs (energy cutoff $130 \mathrm{eV}$ ) where the three thresholds $10^{-9}, 10^{-10}$, and $10^{-14}$ lead to 243,144 , and 60 functions being removed from the auxiliary basis. In this case, threshold $10^{-10}$ produces the best accuracy. When higher energy PWs are included in the MDF expansion, more diffuse Gaussians are removed by the threshold, and the PW functions increasingly take over the role of expanding the diffuse density. The different thresholds produce similar convergence. When the PW functions and Gaussians are overlapped for the expansion of the valence density, corresponding to the energy cutoff $2000 \mathrm{eV}$ (41 PWs per axis) or higher, the effects of basis incompleteness error are observed for threshold $10^{-9}$ and the numerical instability issue is observed for $10^{-14}$.

As discussed in the Introduction, an important motivation for all-electron calculations enabled by MDF is that they allow us to assess pseudo-potential error. We now briefly examine the pseudo-potential error in the band structure of the silicon crystal. Figure 3 presents the LDA band structure computed within a pseudopotential (PP) and an all-electron calculation using a $6 \times 6 \times 6 k$-point mesh with two atoms per (primitive) unit cell. In the PP calculation, we used the GTH pseudopotentials ${ }^{43,44}$ that were optimized for the LDA

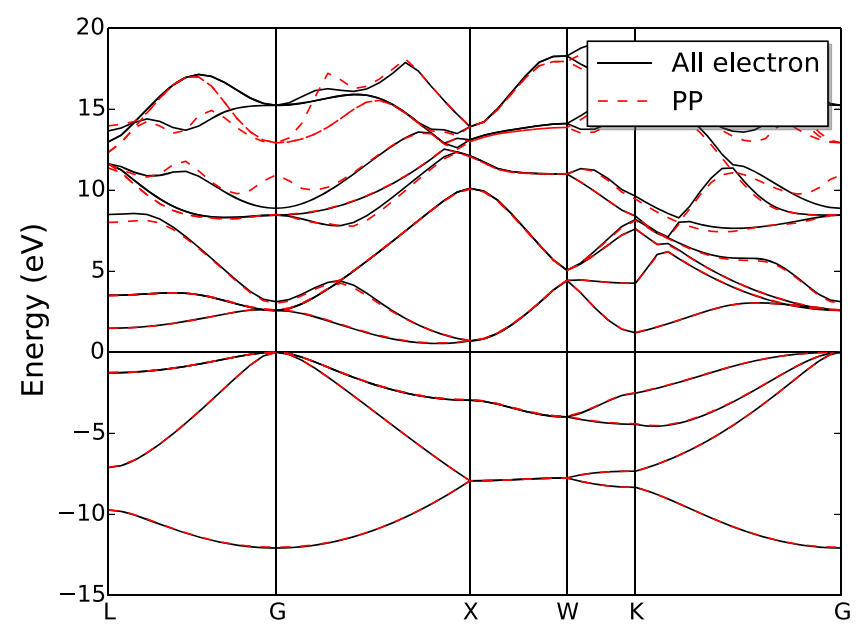

FIG. 3. All-electron and pseudopotential LDA band structure of the Si crystal. functional and the GTH DZVP basis, obtained from the CP2 $\mathrm{K}^{36,45}$ software package. The PP Coulomb integrals were computed with 3375 PWs (energy cutoff $750 \mathrm{eV}$ ) using FFTs. In the all-electron calculation, we used the cc-pVDZ AO basis and a fitting basis consisting of the ETB basis $20 s 16 p 13 d 7 f 2 g$ and $1331 \mathrm{PWs}$ (energy cutoff $380 \mathrm{eV}$ ). The valence bands and conduction bands agree very well between the two types of calculations near the Fermi level although quantitative discrepancies appear further from the Fermi level. The bandgap of the PP calculation is $0.72 \mathrm{eV}$, while the all electron calculation predicts a bandgap of $0.69 \mathrm{eV}$, which is very good agreement considering the slight difference in the single-particle basis set.

Finally, we briefly compare the computational cost of the MDF method in all-electron and pseudo-potential calculations to our earlier Gaussian orbital FFT-based pseudo-potential algorithm. ${ }^{41,42}$ In the FFT-based DFT calculation, evaluating the Gaussian $\mathrm{AO}$ values on the real-space grid is the expensive operation with a formal scaling of $O\left(n N_{G} N_{c}\right)$. As shown in Sec. II A, the scaling of the AFT in the MDF integrals is $n$ times higher than the scaling of AO evaluation. In addition to the analytical Fourier transforms, the MDF method also requires the three-index Gaussian integrals, and these are computationally demanding as well. In the applications to the silicon crystal test system above using pseudopotentials, we found that the cost of the pseudo-potential MDF calculation was about an order of magnitude higher than the pseudopotential FFT-based calculation. This reflects the fact that the Gaussian fitting basis is not really required to represent purely smooth densities. However, the strength of the MDF procedure is to enable all-electron calculations, and the all-electron calculations using the pure FFT algorithm would be prohibitively (orders of magnitude more) expensive than with the MDF implementation.

\section{CONCLUSIONS}

In this work, we presented a Gaussian and plane-wave mixed density fitting (MDF) to compute electron repulsion integrals and associated quantities such as the Coulomb and exchange energies in periodic systems. Our algorithm possesses several new features, including the use of analytical Fourier transforms instead of the standard fast Fourier Transform to achieve high accuracy, and an efficient transformation to remove linear dependencies between Gaussians and PWs.

MDF allows for periodic calculations both with pseudopotentials and with all electrons. Compared with conventional GDF, the main advantage of MDF is the ability to systematically converge to high accuracy through the PW part of the expansion with a relatively weak dependence on the quality of the Gaussian fitting basis and without the need for diffuse Gaussian fitting functions. The main disadvantage of the technique is the overhead incurred from handling the (relatively) large PW fitting basis. This means that the MDF approach is unlikely to be the method of choice for low-accuracy or pseudo-potential calculations. However, for high-accuracy all-electron calculations in large systems, MDF provides an 
efficient computational choice. Further, it is possible to accelerate MDF calculations by exploiting the dual sparsity of the densities in real and reciprocal space. These optimizations will be considered in our future work.

\section{ACKNOWLEDGMENTS}

Q.S., J.D.M., and G.K.-L.C. were supported by the U.S. Department of Energy (DOE) Grant No. DE-SC0008624 and the NSF Grant No. NSF-SSI: OAC 1657286. Additional support was provided by the Simons Foundation, via the Collaboration on the Many-Electron Problem, and a Simons Investigatorship in Theoretical Physics. T.C.B. was supported by the Princeton Center for Theoretical Science and by start-up funds from the University of Chicago.

\section{APPENDIX A: ANALYTICAL INTEGRALS FOR PERIODIC GAUSSIAN FUNCTIONS}

The integrals we presented in Sec. II A can be evaluated analytically with real space lattice sums. For a crystalmomentum-conserving AO basis $\left(\mathbf{k}_{\mu}=\mathbf{k}_{v}\right)$, the AO overlap integrals (per unit cell) can be computed as

$$
\begin{aligned}
S_{\mu \nu}= & \frac{1}{N}\left\langle\phi_{\mu} \mid \phi_{\nu}\right\rangle \\
= & \frac{1}{N} \int \sum_{\mathbf{T}_{\mu}} e^{-i \mathbf{k}_{\mu} \cdot \mathbf{T}_{\mu}} \mu^{*}\left(\mathbf{r}-\mathbf{T}_{\mu}\right) \sum_{\mathbf{T}_{\nu}} e^{i \mathbf{k}_{\nu} \cdot \mathbf{T}_{\nu}} v\left(\mathbf{r}-\mathbf{T}_{\nu}\right) d \mathbf{r} \\
= & \frac{1}{N} \int \sum_{\mathbf{T}_{\mu}} e^{-i \mathbf{k}_{\mu} \cdot \mathbf{T}_{\mu}} \mu^{*}\left(\mathbf{r}-\mathbf{T}_{\mu}\right) \\
& \times \sum_{\mathbf{T}_{\nu}} e^{i \mathbf{k}_{v} \cdot\left(\mathbf{T}_{\mu}+\mathbf{T}_{\nu}\right)} v\left(\mathbf{r}-\mathbf{T}_{\mu}-\mathbf{T}_{\nu}\right) d \mathbf{r} \\
= & \frac{1}{N} \sum_{\mathbf{T}_{\mu}} e^{i\left(\mathbf{k}_{\nu}-\mathbf{k}_{\mu}\right) \cdot \mathbf{T}_{\mu}} \int \sum_{\mathbf{T}_{\nu}} \mu^{*}(\mathbf{r}) e^{i \mathbf{k}_{\nu} \cdot \mathbf{T}_{\nu}} v\left(\mathbf{r}-\mathbf{T}_{\nu}\right) d \mathbf{r} \\
= & \int \sum_{\mathbf{T}_{\nu}} \mu^{*}(\mathbf{r}) e^{i \mathbf{k}_{\nu} \cdot \mathbf{T}_{\nu}} v\left(\mathbf{r}-\mathbf{T}_{\nu}\right) d \mathbf{r} .
\end{aligned}
$$

A similar treatment can be used for the other two-electron Gaussian integrals,

$$
\begin{aligned}
& \left(\phi_{P}^{-\mathbf{k}} \mid \phi_{Q}^{\mathbf{k}}\right)=\int \phi_{P}^{-\mathbf{k}}\left(\mathbf{r}_{1}\right) \frac{1}{r_{12}} \phi_{Q}^{\mathbf{k}}\left(\mathbf{r}_{2}\right) d \mathbf{r}_{1} \mathbf{r}_{2} \\
& =\sum_{\mathbf{T}} e^{i \mathbf{k} \cdot \mathbf{T}} \int\left[\chi_{P}\left(\mathbf{r}_{1}\right)-\xi_{P}\left(\mathbf{r}_{1}\right)\right] \frac{1}{r_{12}} \\
& \times\left[\chi_{Q}\left(\mathbf{r}_{2}-\mathbf{T}\right)-\xi_{Q}\left(\mathbf{r}_{2}-\mathbf{T}\right)\right] d \mathbf{r}_{1} d \mathbf{r}_{2}, \\
& \left(\phi_{P}^{-\mathbf{k}_{\kappa \lambda}} \mid \phi_{\mu}^{\mathbf{k}_{\mu} *} \phi_{v}^{\mathbf{k}_{v}}\right)=\frac{1}{\sqrt{N}} \int \phi_{P}^{-\mathbf{k}_{\kappa \lambda}}\left(\mathbf{r}_{1}\right) \frac{1}{r_{12}} \phi_{\mu}^{\mathbf{k}_{\mu} *}\left(\mathbf{r}_{2}\right) \phi_{v}^{\mathbf{k}_{\nu}}\left(\mathbf{r}_{2}\right) d \mathbf{r}_{1} d \mathbf{r}_{2} \\
& =\sum_{\mathbf{T}_{\mu} \mathbf{T}_{\nu}} e^{i \mathbf{k}_{\nu} \cdot \mathbf{T}_{\nu}-i \mathbf{k}_{\mu} \cdot \mathbf{T}_{\mu}} \int\left[\chi_{P}\left(\mathbf{r}_{1}\right)-\xi_{P}\left(\mathbf{r}_{1}\right)\right] \\
& \times \frac{1}{r_{12}} \mu^{*}\left(\mathbf{r}_{2}-\mathbf{T}_{\mu}\right) v\left(\mathbf{r}_{2}-\mathbf{T}_{v}\right) d \mathbf{r}_{1} d \mathbf{r}_{2},
\end{aligned}
$$

and Fourier transforms,

$$
\begin{aligned}
& \rho_{P}(\mathbf{G}+\mathbf{k})=\frac{1}{\sqrt{N}} \int e^{-i(\mathbf{G}+\mathbf{k}) \cdot \mathbf{r}} \phi_{P}^{\mathbf{k}}(\mathbf{r}) d \mathbf{r} \\
& =\frac{1}{N} \int e^{-i(\mathbf{G}+\mathbf{k}) \cdot \mathbf{r}} \sum_{\mathbf{T}} e^{i \mathbf{k} \cdot \mathbf{T}}\left[\chi_{P}(\mathbf{r}-\mathbf{T})-\xi_{P}(\mathbf{r}-\mathbf{T})\right] d \mathbf{r} \\
& =\frac{1}{N} \int \sum_{\mathbf{T}} e^{-i(\mathbf{G}+\mathbf{k}) \cdot(\mathbf{r}+\mathbf{T})} e^{i \mathbf{k} \cdot \mathbf{T}}\left[\chi_{P}(\mathbf{r})-\xi_{P}(\mathbf{r})\right] d \mathbf{r} \\
& =\int e^{-i(\mathbf{G}+\mathbf{k}) \cdot \mathbf{r}}\left[\chi_{P}(\mathbf{r})-\xi_{P}(\mathbf{r})\right] d \mathbf{r}, \\
& \rho_{\mu \nu}\left(\mathbf{G}+\mathbf{k}_{\mu \nu}\right)=\frac{1}{N} \int e^{-i\left(\mathbf{G}+\mathbf{k}_{\mu \nu}\right) \cdot \mathbf{r}} \phi_{\mu}^{*}(\mathbf{r}) \phi_{\nu}(\mathbf{r}) d \mathbf{r} \\
& =\frac{1}{N} \int e^{-i\left(\mathbf{G}+\mathbf{k}_{\mu \nu}\right) \cdot \mathbf{r}} \sum_{\mathbf{T}_{\mu}} e^{-i \mathbf{k}_{\mu} \cdot \mathbf{T}_{\mu}} \mu^{*}\left(\mathbf{r}-\mathbf{T}_{\mu}\right) \\
& \times \sum_{\mathbf{T}_{v}} e^{i \mathbf{k}_{v} \cdot \mathbf{T}_{v}} v\left(\mathbf{r}-\mathbf{T}_{v}\right) d \mathbf{r} \\
& =\frac{1}{N} \int \sum_{\mathbf{T}_{\mu}} e^{-i\left(\mathbf{G}+\mathbf{k}_{\mu \nu}\right) \cdot\left(\mathbf{r}+\mathbf{T}_{\mu}\right)} e^{-i \mathbf{k}_{\mu} \cdot \mathbf{T}_{\mu}} \mu^{*}(\mathbf{r}) \\
& \times \sum_{\mathbf{T}_{v}} e^{i \mathbf{k}_{v} \cdot \mathbf{T}_{v}} v\left(\mathbf{r}+\mathbf{T}_{\mu}-\mathbf{T}_{v}\right) d \mathbf{r} \\
& =\frac{1}{N} \int \sum_{\mathbf{T}_{\mu}} e^{-i\left(\mathbf{G}+\mathbf{k}_{\mu \nu}\right) \cdot\left(\mathbf{r}+\mathbf{T}_{\mu}\right)} e^{-i \mathbf{k}_{\mu} \cdot \mathbf{T}_{\mu}} \mu^{*}(\mathbf{r}) \\
& \times \sum_{\mathbf{T}_{\nu}} e^{i \mathbf{k}_{\nu} \cdot\left(\mathbf{T}_{\mu}+\mathbf{T}_{\nu}\right)} v\left(\mathbf{r}-\mathbf{T}_{\nu}\right) d \mathbf{r} \\
& =\sum_{\mathbf{T}_{v}} e^{i \mathbf{k}_{\nu} \cdot \mathbf{T}_{v}} \int e^{-i\left(\mathbf{G}+\mathbf{k}_{\mu \nu}\right) \cdot \mathbf{r}} \mu^{*}(\mathbf{r}) v\left(\mathbf{r}-\mathbf{T}_{v}\right) d \mathbf{r} .
\end{aligned}
$$

Integral (14) is computed as

$$
\begin{aligned}
\bar{V}_{P}^{\mathbf{k}} & =\lim _{N \rightarrow \infty} \frac{1}{\sqrt{N}} \int \frac{\phi_{Q}^{\mathbf{k}}\left(\mathbf{r}_{2}\right)}{r_{12}} d \mathbf{r}_{1} d \mathbf{r}_{2} \\
& =\lim _{N \rightarrow \infty} \frac{1}{N} \sum_{\mathbf{T}} e^{i \mathbf{k} \cdot \mathbf{T}} \int \frac{\chi_{P}\left(\mathbf{r}_{2}\right)-\xi_{P}\left(\mathbf{r}_{2}\right)}{r_{12}} d \mathbf{r}_{1} d \mathbf{r}_{2} .
\end{aligned}
$$

The limits of this integral are non-vanishing only if $\mathbf{k}=0$ and the integrands $\chi_{P}$ and $\xi_{P}$ are of $s$-type spherical symmetry,

$$
\begin{aligned}
\bar{V}_{P}^{\mathbf{k}}= & \int \frac{1}{r_{12}}\left[\left(\frac{\alpha_{P_{\chi}}}{\pi}\right)^{3 / 2} e^{-\alpha_{P_{\chi}}\left|\mathbf{r}_{2}-\mathbf{R}\right|^{2}}\right. \\
& \left.-\left(\frac{\alpha_{P_{\xi}}}{\pi}\right)^{3 / 2} e^{-\alpha_{P_{\xi}}\left|\mathbf{r}_{2}-\mathbf{R}\right|^{2}}\right] d \mathbf{r}_{1} d \mathbf{r}_{2} \\
= & \frac{1}{(2 \pi)^{3}} \iint e^{i \mathbf{G} \cdot \mathbf{r}_{1}} d \mathbf{r}_{1} \frac{4 \pi}{G^{2}} \\
& \times\left(e^{-\frac{G^{2}}{4 \alpha_{P}}} e^{-i \mathbf{G} \cdot \mathbf{R}}-e^{-\frac{G^{2}}{4 \alpha_{P_{\xi}}}} e^{-i \mathbf{G} \cdot \mathbf{R}}\right) d \mathbf{G} \\
= & \int \delta(\mathbf{G}) \frac{4 \pi}{G^{2}}\left(e^{-\frac{G^{2}}{4 \alpha_{P_{\chi}}}}-e^{-\frac{G^{2}}{4 \alpha_{P_{\xi}}}}\right) e^{-i \mathbf{G} \cdot \mathbf{R}} d \mathbf{G} \\
= & \lim _{G \rightarrow 0} \frac{4 \pi}{G^{2}} \frac{e^{-\frac{G^{2}}{4 \alpha_{P_{\chi}}}}-e^{-\frac{G^{2}}{4 \alpha_{P_{\xi}}}}}{e^{i \mathbf{G} \cdot \mathbf{R}}} \\
= & \frac{\pi}{\alpha_{P_{\xi}}}-\frac{\pi}{\alpha_{P_{\chi}}} .
\end{aligned}
$$




\section{APPENDIX B: ANALYTICAL FOURIER TRANSFORMATION}

We applied analytical Fourier transformations in this work to guarantee the accuracy of the two-electron integrals. Given Gaussian functions

$$
\begin{aligned}
& \mu(\mathbf{r})=C_{\mu}\left(x-R_{x \mu}\right)^{m_{x}}\left(y-R_{y \mu}\right)^{m_{y}}\left(z-R_{z \mu}\right)^{m_{z}} e^{-\alpha_{\mu}\left|\mathbf{r}-\mathbf{R}_{\mu}\right|^{2},} \\
& v(\mathbf{r})=C_{v}\left(x-R_{x v}\right)^{n_{x}}\left(y-R_{y v}\right)^{n_{y}}\left(z-R_{z v}\right)^{n_{z}} e^{-\alpha_{v}\left|\mathbf{r}-\mathbf{R}_{\nu}\right|^{2},}
\end{aligned}
$$

analytical Fourier transformations for the Gaussian function products can be computed as the products of three Cartesian components,

$$
\begin{aligned}
& \int e^{-i \mathbf{G} \cdot \mathbf{r}} \mu^{*}(\mathbf{r}) v(\mathbf{r}) d \mathbf{r}=C_{\mu} C_{\nu} I_{m_{x}, n_{x}}^{x} I_{m_{y}, n_{y}}^{y} I_{m_{z}, n_{z}}^{z}, \\
I_{m_{x}, n_{x}}^{x}= & \int e^{-i G_{x} x}\left(x-R_{x \mu}\right)^{m_{x}} e^{-\alpha_{\mu}\left(x-R_{x \mu}\right)^{2}}\left(x-R_{x v}\right)^{n_{x}} \\
& \times e^{-\alpha_{\nu}\left(x-R_{x \mu}\right)^{2}} d x .
\end{aligned}
$$

Each Cartesian component can be evaluated through the recursive relations

$$
\begin{aligned}
I_{0,0}^{x} & =\sqrt{\frac{\pi}{\alpha_{\mu}+\alpha_{\nu}}} e^{-\frac{\alpha_{\mu} \alpha_{\nu}}{\alpha_{\mu}+\alpha_{\nu}}\left(R_{x \mu}-R_{x \nu}\right)^{2}} e^{-\frac{G_{x}^{2}}{4\left(\alpha_{\mu}+\alpha_{\nu}\right)}} e^{-i G_{x} X_{\mu \nu}}, \\
I_{1,0}^{x} & =-\left(R_{x \mu}-X_{\mu \nu}+\frac{i G_{x}}{2\left(\alpha_{\mu}+\alpha_{v}\right)}\right) I_{0,0}^{x}, \\
I_{m_{x}, 0}^{x} & =\frac{m_{x}-1}{2\left(\alpha_{\mu}+\alpha_{\nu}\right)} I_{m_{x}-2,0}^{x}-\left(R_{x \mu}-X_{\mu \nu}+\frac{i G_{x}}{2\left(\alpha_{\mu}+\alpha_{\nu}\right)}\right) I_{m_{x}-1,0}^{x}, \\
I_{m_{x}, n_{x}}^{x} & =I_{m_{x}+1, n_{x}-1}^{x}+\left(R_{x \mu}-R_{x \nu}\right) I_{m_{x}, n_{x}-1}^{x},
\end{aligned}
$$

where

$$
X_{\mu v}=\frac{\alpha_{\mu} R_{x \mu}+\alpha_{v} R_{x v}}{\alpha_{\mu}+\alpha_{v}}
$$

${ }^{1}$ J. L. Whitten, J. Chem. Phys. 58, 4496 (1973).

${ }^{2}$ B. I. Dunlap, J. W. D. Connolly, and J. R. Sabin, J. Chem. Phys. 71, 3396 (1979).

${ }^{3}$ O. Vahtras, J. Almlöf, and M. Feyereisen, Chem. Phys. Lett. 213, 514 (1993).

${ }^{4}$ K. Eichkorn, O. Treutler, H. Öhm, M. Häser, and R. Ahlrichs, Chem. Phys. Lett. 240, 283 (1995).

${ }^{5}$ B. I. Dunlap, Phys. Chem. Chem. Phys. 2, 2113 (2000).

${ }^{6}$ G. te Velde and E. J. Baerends, Phys. Rev. B 44, 7888 (1991).

${ }^{7}$ J. E. Jaffe and A. C. Hess, J. Chem. Phys. 105, 10983 (1996).

${ }^{8}$ P. Čársky, R. Čurík, and Š. Varga, J. Chem. Phys. 136, 114105 (2012).

${ }^{9}$ A. M. Burow, M. Sierka, and F. Mohamed, J. Chem. Phys. 131, 214101 (2009).

${ }^{10}$ M. D. Ben, J. Hutter, and J. VandeVondele, J. Chem. Theory Comput. 9, 2654 (2013).
${ }^{11}$ C. Pisani, L. Maschio, S. Casassa, M. Halo, M. Schütz, and D. Usvyat, J. Comput. Chem. 29, 2113 (2008).

${ }^{12}$ L. Maschio, D. Usvyat, F. R. Manby, S. Casassa, C. Pisani, and M. Schütz, Phys. Rev. B 76, 075101 (2007).

${ }^{13}$ D. Usvyat, L. Maschio, F. R. Manby, S. Casassa, M. Schütz, and C. Pisani, Phys. Rev. B 76, 075102 (2007).

${ }^{14}$ L. Maschio and D. Usvyat, Phys. Rev. B 78, 073102 (2008).

${ }^{15}$ B. I. Dunlap, N. Rösch, and S. Trickey, Mol. Phys. 108, 3167 (2010).

${ }^{16}$ N. H. F. Beebe and J. Linderberg, Int. J. Quantum Chem. 12, 683 (1977).

${ }^{17}$ I. Røeggen and E. Wisløff-Nilssen, Chem. Phys. Lett. 132, 154 (1986).

${ }^{18}$ F. Aquilante, L. Gagliardi, T. B. Pedersen, and R. Lindh, J. Chem. Phys. 130, 154107 (2009).

${ }^{19}$ L. Füsti-Molnar and P. Pulay, J. Chem. Phys. 116, 7795 (2002).

${ }^{20}$ L. Füsti-Molnar, J. Chem. Phys. 119, 11080 (2003).

${ }^{21}$ G. Lippert, J. Hutter, and M. Parrinello, Mol. Phys. 92, 477 (1997).

${ }^{22}$ G. Lippert, J. Hutter, and M. Parrinello, Theor. Chem. Acc. 103, 124 (1999).

${ }^{23}$ L. Füsti-Molnar and J. Kong, J. Chem. Phys. 122, 074108 (2005).

${ }^{24}$ R. B. Murphy, Y. Cao, M. D. Beachy, M. N. Ringnalda, and R. A. Friesner, J. Chem. Phys. 112, 10131 (2000).

${ }^{25}$ F. Neese, F. Wennmohs, A. Hansen, and U. Becker, Chem. Phys. 356, 98 (2009).

${ }^{26}$ M. Feyereisen, G. Fitzgerald, and A. Komornicki, Chem. Phys. Lett. 208, 359 (1993).

${ }^{27}$ A. Komornicki and G. Fitzgerald, J. Chem. Phys. 98, 1398 (1993).

${ }^{28}$ F. Weigend, M. Häser, H. Patzelt, and R. Ahlrichs, Chem. Phys. Lett. 294, 143 (1998).

${ }^{29}$ M. Sierka, A. Hogekamp, and R. Ahlrichs, J. Chem. Phys. 118, 9136 (2003).

${ }^{30}$ F. R. Manby, P. J. Knowles, and A. W. Lloyd, J. Chem. Phys. 115, 9144 (2001).

${ }^{31}$ H.-J. Werner, F. R. Manby, and P. J. Knowles, J. Chem. Phys. 118, 8149 (2003).

${ }^{32}$ A. Sodt, J. E. Subotnik, and M. Head-Gordon, J. Chem. Phys. 125, 194109 (2006).

${ }^{33}$ H.-J. Werner and F. R. Manby, J. Chem. Phys. 124, 054114 (2006).

${ }^{34}$ Y. Jung, A. Sodt, P. M. W. Gill, and M. Head-Gordon, Proc. Natl. Acad. Sci. U. S. A. 102, 6692 (2005), http://www.pnas.org/content/ 102/19/6692.full.pdf.

${ }^{35}$ M. Krack and M. Parrinello, Phys. Chem. Chem. Phys. 2, 2105 (2000).

${ }^{36}$ J. VandeVondele, M. Krack, F. Mohamed, M. Parrinello, T. Chassaing, and J. Hutter, Comput. Phys. Commun. 167, 103 (2005).

${ }^{37}$ L. Füsti-Molnar and P. Pulay, J. Chem. Phys. 117, 7827 (2002).

${ }^{38}$ R. Dovesi, B. Civalleri, C. Roetti, V. R. Saunders, and R. Orlando, "Ab initio quantum simulation in solid state chemistry," in Reviews in Computational Chemistry (John Wiley \& Sons, Inc., 2005), pp. 1-125.

${ }^{39}$ J. Kolafa and J. W. Perram, Mol. Simul. 9, 351 (1992).

${ }^{40} \mathrm{P}$. Gibbon and G. Sutmann, Lecture Notes, NIC Series, edited by J. Grotendorst, D. Marx, and A. Muramatsu (John von Neumann Institute for Computing, Jülich, 2002), pp. 467-506.

${ }^{41}$ J. McClain, Q. Sun, G. K.-L. Chan, and T. C. Berkelbach, J. Chem. Theory Comput. 13, 1209 (2017).

${ }^{42}$ Q. Sun, T. C. Berkelbach, N. S. Blunt, G. H. Booth, S. Guo, Z. Li, J. Liu, J. McClain, E. R. Sayfutyarova, S. Sharma, S. Wouters, and G. K.-L. Chan, "PySCF: The Python-based simulations of chemistry framework," Wiley Interdiscip. Rev.: Comput. Mol. Sci. (published online).

${ }^{43}$ S. Goedecker, M. Teter, and J. Hutter, Phys. Rev. B 54, 1703 (1996).

${ }^{44}$ C. Hartwigsen, S. Goedecker, and J. Hutter, Phys. Rev. B 58, 3641 (1998).

${ }^{45}$ J. Hutter, M. Iannuzzi, F. Schiffmann, and J. VandeVondele, Wiley Interdiscip. Rev.: Comput. Mol. Sci. 4, 15 (2014). 